\title{
The production of graphene-boron nitride nanosheet heterostructures via liquid phase exfoliation assisted by a milling process
}

\author{
ÖMER GÜLER*, SEVAL H GÜLER and MUSTAFA TAŞKIN \\ Metalurgical and Material Engineering Department, Mersin University, Mersin, Turkey \\ *Author for correspondence (oguler@mersin.edu.tr)
}

MS received 6 December 2017; accepted 26 May 2018; published online 8 January 2019

\begin{abstract}
Graphene-boron nitride (BN) nanosheet heterostructures have become one of the highly interesting matters in recent times owing to their advantages. In this study, the liquid phase exfoliation method was preferred for production of graphene-BN nanosheet heterostructures. However, a pre-milling process was applied to starting materials, instead of the classical liquid phase exfoliation method previously used in the literature. Hexagonal graphite (h-G) and h-BN mixtures were milled for $50 \mathrm{~h}$ and the milled powders were subjected to the liquid phase exfoliation process. As a result of the examinations, it was observed that graphene-BN nanosheet heterostructures were successfully synthesized. The widths of the synthesized nanosheets were 300-500 nm and nanosheets were multi-layers. It was seen that a large part of the powder mixture were occurred at high amorphization during the ball milling process. According to X-ray diffraction (XRD) peaks, the amorphization ratio was almost $90 \%$. But, almost all of amorphous structures were removed during acid mixing and the thermal process. But, amorphous structures still existed in samples. In addition, the ball milling process damaged the sheets and defects formed. Despite all these disadvantages, the milling process carried out in this study provided formation of thinner and larger sheets compared with previous similar studies.
\end{abstract}

Keywords. Graphene; BN nanosheets; heterostructures; exfoliation; ball milling.

\section{Introduction}

Graphene is a two-dimensional allotrope of carbon formed by arraying carbon atoms in the form of a honey-comb lattice. In other words, a single layer of hexagonal graphite (h-G) which has a stratified structure is called graphene [1-3]. After Geim and Novoselov discovered and produced this material in 2004, interest in this material has fabulously increased [4,5]. The reason behind why graphene has become a cynosure in the science world is that it has an extremely high Young's modulus compared with classical materials we have known so far, it has an extremely high specific surface area, exhibits a thermal conductivity two times more than thermal conductivity of diamonds with the highest-known thermal conductivity and shows high electrical conductivity [1,6,7]. These characteristics, which are not seen in classical materials, have allowed graphene to be used as a new generation electronic material. It will be used in especially new generation field-effect transistors, batteries, supercapacitors and sensors in the near future $[8,9]$.

Hexagonal boron nitride (h-BN) also has a structure similar to graphite and it consists of hexagonal $\mathrm{BN}$ sheets that are regularly stacked. Because most characteristics of h$\mathrm{BN}$ are similar to graphite, it is known as 'white graphite'. $\mathrm{BN}$ nanosheets, which are the single layer of h-BN, display characteristics similar to graphene. Because it has a wide band gap $(5-6 \mathrm{eV})$, it is electrically insulating differently from graphene. Due to these properties, they have important potential for their use in optoelectronic applications and heatreleasing composites [10-13].

Graphene has high electron carrier mobility, but it is a zero band gap semimetal. When graphene is used in the $\mathrm{SiO}_{2}$ substrate to manufacture a transistor, performance of graphene decreases due to roughness of the surface, charged surface and $\mathrm{SiO}_{2}$ surface optical phonons. Therefore, graphenebased heterostructures are preferred instead of using pure graphene especially in transistor applications. GrapheneBNNS heterostructures have been widely preferred recently in developing graphene-based transistors. BNNSs have an ultraflat surface, they are free of dangling bonds and they increase performance of graphene in case when they are used as substrates [14-17].

In our previous studies, graphene was produced by using the method of liquid phase exfoliation assisted by a milling process [18]. A graphene-BNNS mixture was successfully produced only by using the liquid phase exfoliation method in our previous study [19]. In this study, on the other hand, producibility of graphene-BNNS heterostructures by a milling process and subsequently by an exfoliation method was investigated. 


\section{Experimental}

h-G (Merck, 99.5\%) and h-BN (Merck, 99.5\%) powders were used as starting materials in this study. h-G and h-BN powders were dried in a furnace at $75^{\circ} \mathrm{C}$ for $2 \mathrm{~h}$. Dehydrated $\mathrm{h}-\mathrm{G}$ and $\mathrm{h}$-BN powders were then weighed at the same rate by weight and subjected to a milling process using $19-\mathrm{mm}$ single ball in separate vessels at $400 \mathrm{rpm}$ for $50 \mathrm{~h}$ in a Fritsch Pulverisette Premium line 7 device. The powders obtained after the milling process were mixed in equal amounts by weight and this mixture was subjected to a two-stage process in order to increase distance between the sheets. In the first process, the powders were mixed using a magnetic stirrer in a saturated acid solution containing $\mathrm{H}_{2} \mathrm{SO}_{4}$ and $\mathrm{HNO}_{3}$ for $12 \mathrm{~h}$ in order to obtain a homogeneous mixture. The powder mixture, which was filtered from acid solution, was washed with distilled water until $\mathrm{pH}$ becomes neutral. The powders obtained at the end of this process were heated at $950^{\circ} \mathrm{C}$ in air as the second stage. Following the thermal process applied in the second stage, an h-G/h-BN powder mixture with increasing distance between the sheets was obtained. Despite the fact that the distance between the sheets of the powder mixture was expanded, sheets were still bound to each other with van der Waals bonds. To obtain graphene-BNNS heterostructures, the powder mixture with expanded distance between the sheets was mixed within $N, N$-dimethylformamide (DMF) with the help of an ultra-sonic homogenizer (Bandelin Sonoplus, model HD 3200, $200 \mathrm{~W}$, at $50 \%$ power) for $2 \mathrm{~h}$. A suspension of $0.05 \mathrm{mg} \mathrm{ml}^{-1}$ concentration of the powder mixture with expanded distance between the sheets by using DMF was prepared. Then, the mixture was centrifuged at $5000 \mathrm{rpm}$ for $8 \mathrm{~h}$ to remove DMF. The powders were washed using ethanol and were dried in a vacuum oven at $75^{\circ} \mathrm{C}$ for $4 \mathrm{~h}$ to remove the alcohol.

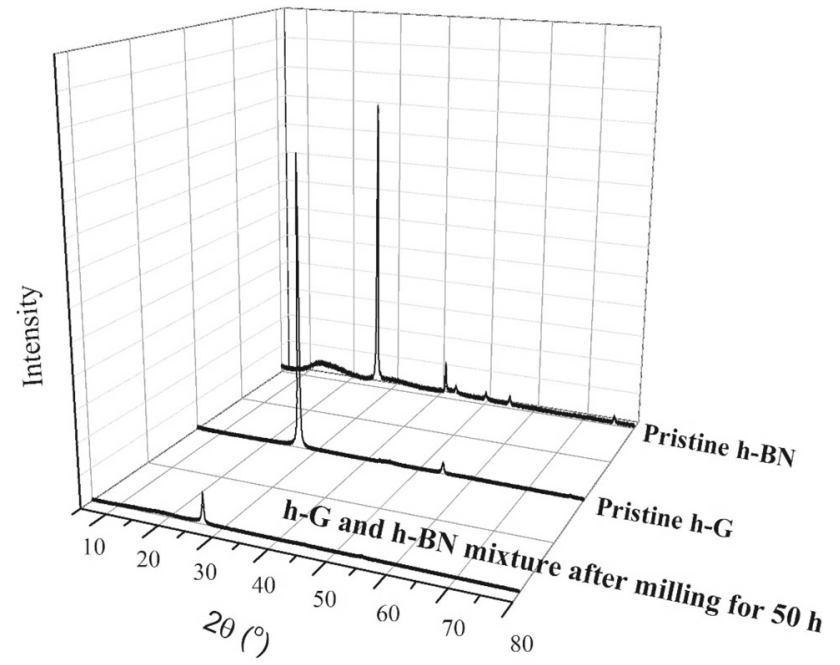

Figure 1. XRD analysis of pristine $\mathrm{h}-\mathrm{G}$, pristine $\mathrm{h}-\mathrm{BN}$ and milled h-G and h-BN mixture.
The obtained powder was characterized by using UV-vis spectroscopy. The spectrum had an operation range (UV 3600 Shimadzu, Japan) between 250 and $1200 \mathrm{~nm}$. High-resolution transmission electron microscopy (HRTEM) (JEOL Jem 2100F) was used to investigate the microstructure of graphene samples. The samples were analysed by using an FESEM (Jeol Jsm-7001F). Additionally, Raman spectroscopy (Witec snom-Raman) was performed by using a laser excitation of $532 \mathrm{~nm}$ delivered through a $100 \times$ objective. Thermogravimetric analysis (TGA) of the samples was carried out under an air atmosphere from room temperature to $700^{\circ} \mathrm{C}$ at a heating rate of $5^{\circ} \mathrm{C} \mathrm{min}^{-1}$ on a Shimadzu TA-60WS device.

\section{Results}

h-G and h-BN have a structure consisting of many stratified sheets. Atoms in sheets were bound to each other with strong bonds. A bond that kept stratified sheets together was a weak
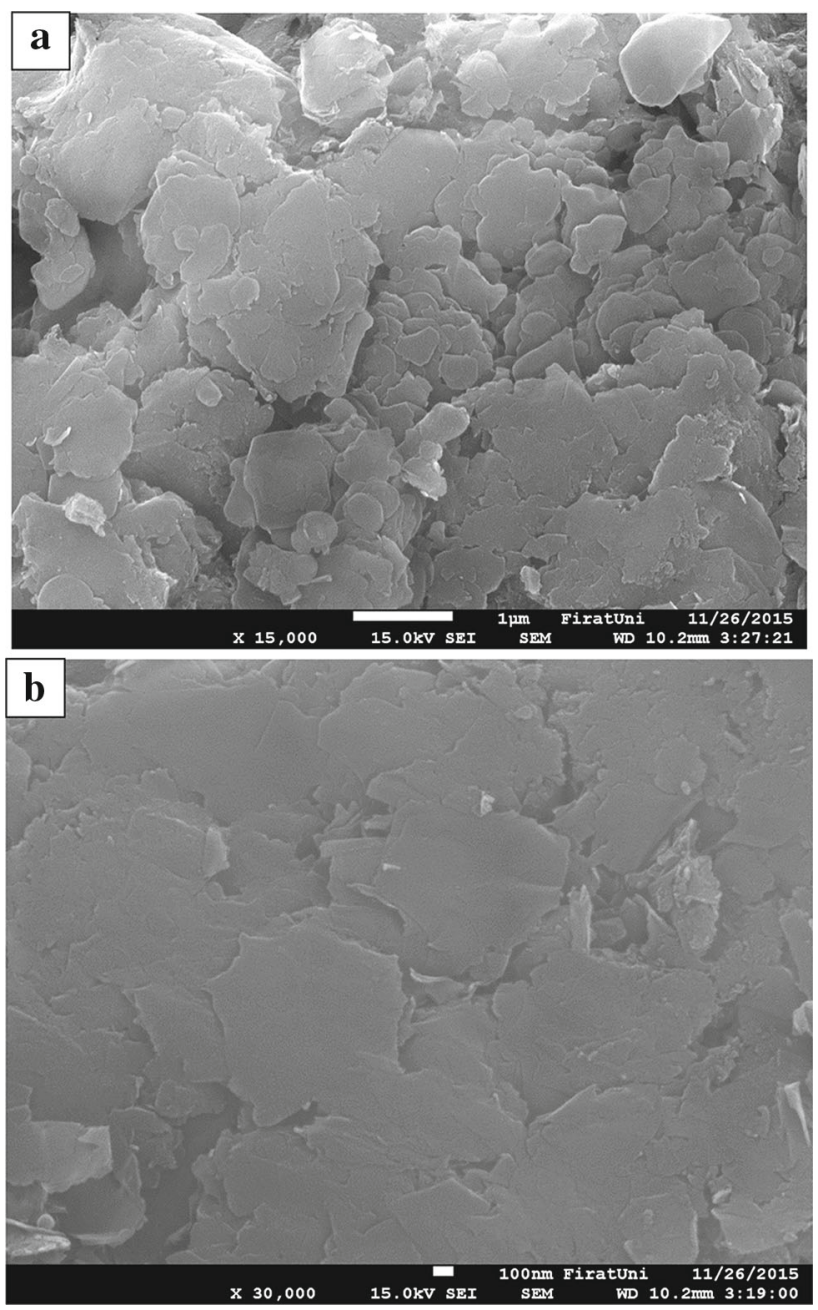

Figure 2. (a, b) SEM micrographs of milled mixture powders after acid treatment. 

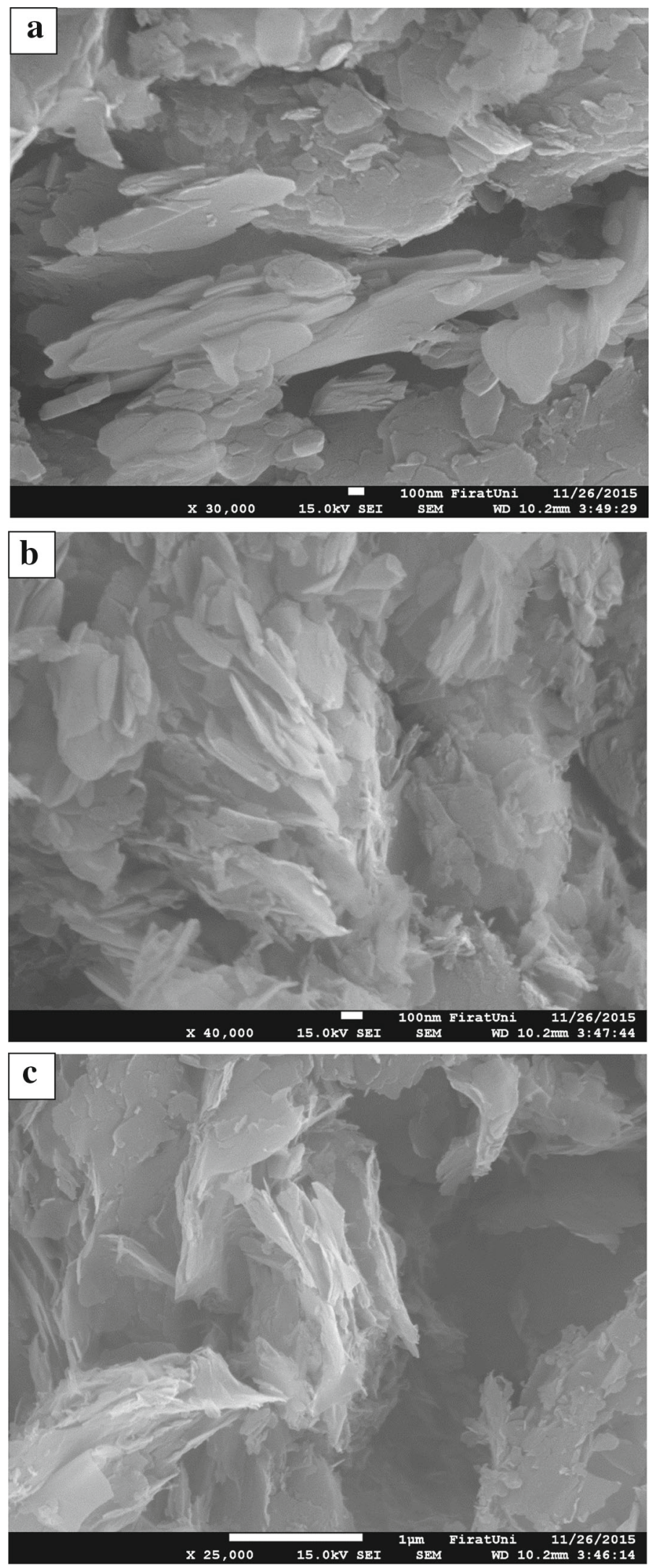

Figure 3. (a-c) SEM micrographs of powders (expanded) after heat treatment. 
bond. The liquid phase exfoliation method is based on making sheets free by rupturing the weak bonds that binds sheets with each other [19-21]. An organic solvent is used to achieve this. The atoms of this organic solvent ensure to break the weak bonds by spreading between the sheets. The distance between the sheets in h-G and h-BN is $0.334 \mathrm{~nm}$ and this distance is not usually enough for the atoms of the organic solvent to enter this space. The amount of nanosheets occurring in the case of liquid phase exfoliation of $\mathrm{h}-\mathrm{G}$ and $\mathrm{h}-\mathrm{BN}$ without subjecting to any pretreatment was very low [21]. It is needed to expand the distance between the h-G and h-BN sheets in order to increase the amount of product. As explained in section 2, powders were mixed in acid and then were subjected to the thermal process in order to increase the distance between the sheets. It was reported in previous studies that nanosheets produced for expanding distance between the sheets of h-G and h-BN were not at the desired amount and some of the formed sheets were relatively thick [22]. In the current study, $\mathrm{h}-\mathrm{G}$ and $\mathrm{h}-\mathrm{BN}$ powders were firstly subjected to a milling process in order to decrease the size of the micro-sized hexagonal mixing sheets before the liquid exfoliation method. Thus, the amount of the final product was aimed to increase with liquidphase exfoliation method to be applied to nanosheets. The milling process carried out in this study was different from conventional milling processes. Shear stresses were effective during the milling process due to the use of a single ball with a large diameter. Impact stresses were at the minimum level [23].

Figure 1 shows X-ray diffraction (XRD) analyses of $h-G$ and $\mathrm{h}-\mathrm{BN}$ before milling process and sample obtained at the end of the milling process. Characteristic peaks at about $26^{\circ}$ and with very high intensity (002) were observed in XRD
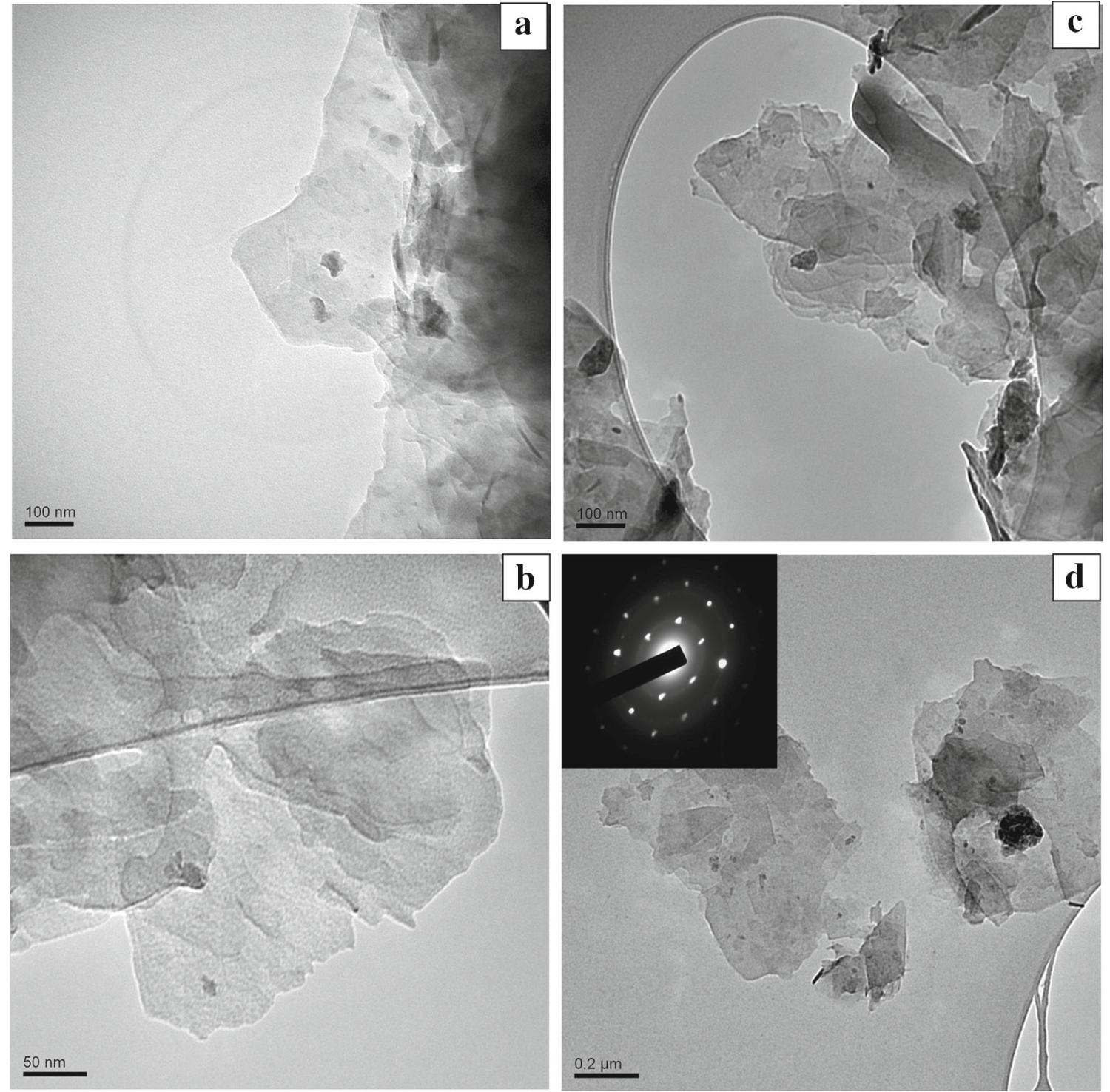

Figure 4. (a-c) TEM micrographs and (d) electron diffraction pattern of synthesized graphene-BN nanosheet heterostructures. 
analyses of both h-G and h-BN. The intensity of the (002) peaks decreased significantly in XRD analyses of the sample obtained after h-G and h-BN powders were subjected to the milling process, in which shear stresses were dominant, and mixing them in equal amounts being most important. The reason for the decrease in intensity of the XRD peaks of sample was because of the impact stresses occurring during the milling process. Even though shear stresses were effective in the milling process, impact stresses occurred as a result of the ball crushing against walls of the vessel. Therefore, powders remaining between the ball and the vessel became amorphous. Despite the intensities of impact stresses occurring during the milling process were low, the milling process of $50 \mathrm{~h}$ increased the amount of amorphization in powders.

A mixture of powders obtained after the milling process was mixed within a saturated acid solution consisting of $\mathrm{H}_{2} \mathrm{SO}_{4}$ and $\mathrm{HNO}_{3}$ for $12 \mathrm{~h}$. Figure 2 shows SEM image of powders filtered at the end of the mixing process and having $\mathrm{a}$ balanced $\mathrm{pH}$. As is seen from figure $2 \mathrm{a}$ and $\mathrm{b}$, powders were in the form of flakes with different widths.

Figure 3, on the other hand, shows SEM image of the sample subjected to the thermal process following acid treatment. Even though there were $\mathrm{h}-\mathrm{G}$ and $\mathrm{h}-\mathrm{BN}$ flakes within the structure (figure 3a), there were expansions between the sheets (figure $3 \mathrm{~b}$ and $\mathrm{c}$ ). In addition, while a regular arrangement of sheets was observed before the process, a regular arrangement of sheets also deformed following the thermal process.

Samples obtained after the thermal process were sonicated within DMF. Figure 4 shows TEM images of graphene-BN nanosheet heterostructures obtained at the end of the sonication process. Figure $4 \mathrm{a}$ and $\mathrm{b}$ shows a few nanometre thick transparent graphene-BN nanosheet heterostructure. In the image, it is possible to observe several stratified nanosheets. It can be asserted that there were numerous defects in the structure when the edges of these sheets were observed. These defects occurred as a result of low impact stresses during the milling process. In addition, as can be seen from figure $4 \mathrm{c}$ and $\mathrm{d}$, there were black non-transparent particles inside the structure and partially on the sheets. These amorphous carbon structures remained inside powders without changing in further stages of experiments.

Figure $4 \mathrm{~d}$ shows graphene-BN nanosheets occurring at the end of experiments and their electron diffraction patterns. These patterns represent diffraction patterns representing a hexagonal structure. Graphene and h-BN have similar lattice constants, and they both appear very similar in the diffraction pattern. In the diffraction pattern of figure $4 \mathrm{~d}$, there are 12 spots, 6 of which belong to graphene and the other 6 belong to the h-BN layer. The existence of points with different sizes and brightness is resulted from the overlapping sheets and/or

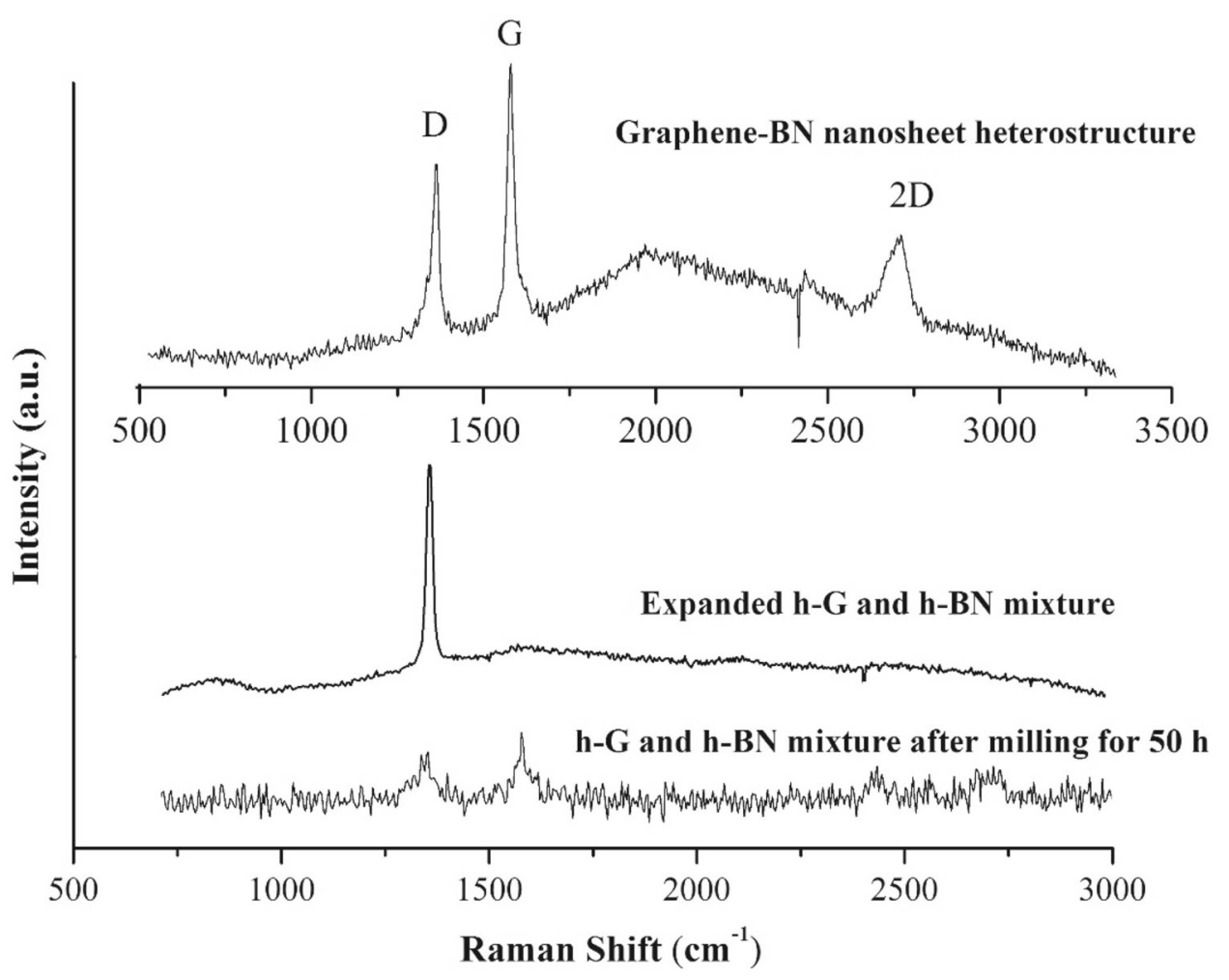

Figure 5. Raman spectroscopy analysis of $\mathrm{h}-\mathrm{G}$ and $\mathrm{h}-\mathrm{BN}$ mixture after milling, expanded $\mathrm{h}-\mathrm{G}$ and h-BN mixture and graphene-BN nanosheet heterostructures. 
coexistence of graphene sheets with BN sheets. Consequently, this diffraction pattern also confirms the existence of graphene and boron sheets in the structure.

Raman spectroscopy is a good method for distinguishing ordered crystal and disordered crystal structures of carbon and BN. In addition, it is also useful for analysing thickness of nanosheets [22]. Carbon materials have three characteristic peaks in the Raman spectrum: D band at $\sim 1350 \mathrm{~cm}^{-1}$, G band at $\sim 1582 \mathrm{~cm}^{-1}$ and $2 \mathrm{D}$ band $\sim 2700 \mathrm{~cm}^{-1}$. Nano-sized graphene sheets show two characteristic bands in the Raman spectrum: a $\mathrm{G}$ band (represents $\mathrm{sp}^{2}$ carbon bonds) due to $\mathrm{E}_{2 \mathrm{~g}}$ phonons at the Brillouin zone centre and a $\mathrm{D}$ band corresponding to the breathing mode of $k$-point phonons at $\mathrm{A}_{1 \mathrm{~g}}$ symmetry [21]. A characteristic peak at $\sim 1366 \mathrm{~cm}^{-1}$ was observed in the Raman spectrum of BN nanosheets. This peak might be associated with high frequency intralayer $\mathrm{E}_{2 \mathrm{~g}}$ vibration mode of $h-B N$. Figure 5 shows the Raman spectrum of the $h-G$ and h-BN mixture milled for $50 \mathrm{~h}, \mathrm{~h}-\mathrm{G}$ and h-BN where distance between sheets was expanded and graphene-BN nanosheet heterostructure. As can be seen from the figure, intensity of all characteristic peaks of powder mixture obtained at the end of the milling process considerably decreased and even peaks were about to disappear. This indicated that amorphization occurred during the milling process and there was little amount of powder with a crystal structure. A significant increase was observed in the peak at $\sim 1360 \mathrm{~cm}^{-1}$ as a result of the two-stage process carried out to increase distance between the sheets of h-G and h-BN. This indicated that the amorphous structures in the sample were removed during the two-stage process. Following sonication of $\mathrm{h}-\mathrm{G}$ and $\mathrm{h}-\mathrm{BN}$, where distance between the sheets was increased, in DMF, $\mathrm{G}$ and 2D bands also occurred [24,25]. Additionally, D band represents $\mathrm{BN}$ nanosheet structures and also it is an indicator of disordered formations in the structure. Partial errors occurring at the edges of graphene and $\mathrm{BN}$ sheets by the impact of ball during the milling process might have caused an increase in this peak [18].

There were a considerable decrease occurring in characteristic peaks in the Raman spectra at the end of the milling process and an increase in characteristic peaks in the Raman spectra of the expanded sample and samples sonicated in DMF. This showed that the amorphous structures forming
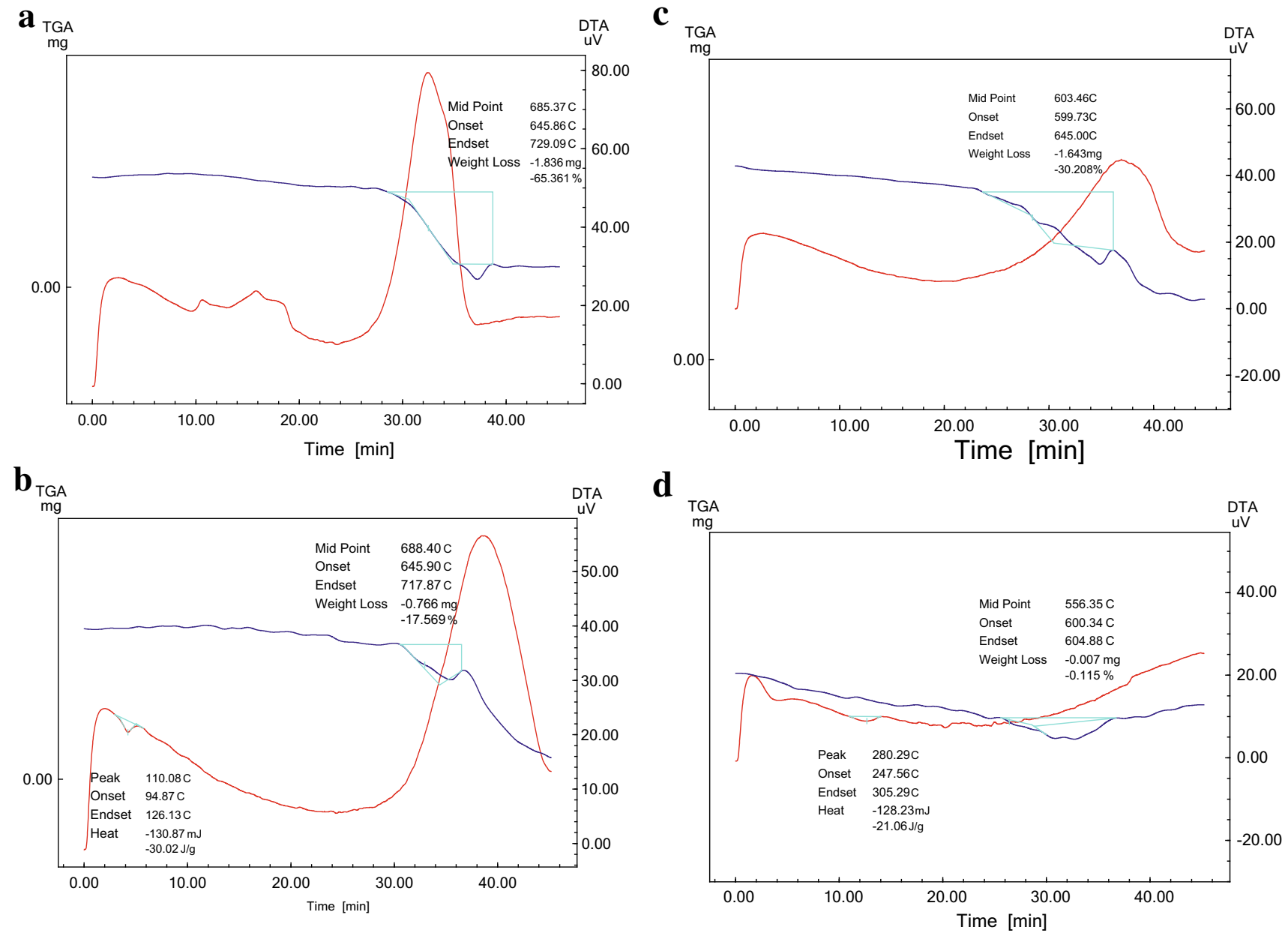

Figure 6. TGA analysis of (a) milled h-G, (b) milled h-BN, (c) h-G and h-BN mixture after acid treatment and (d) expanded h-G and h-BN mixture. 


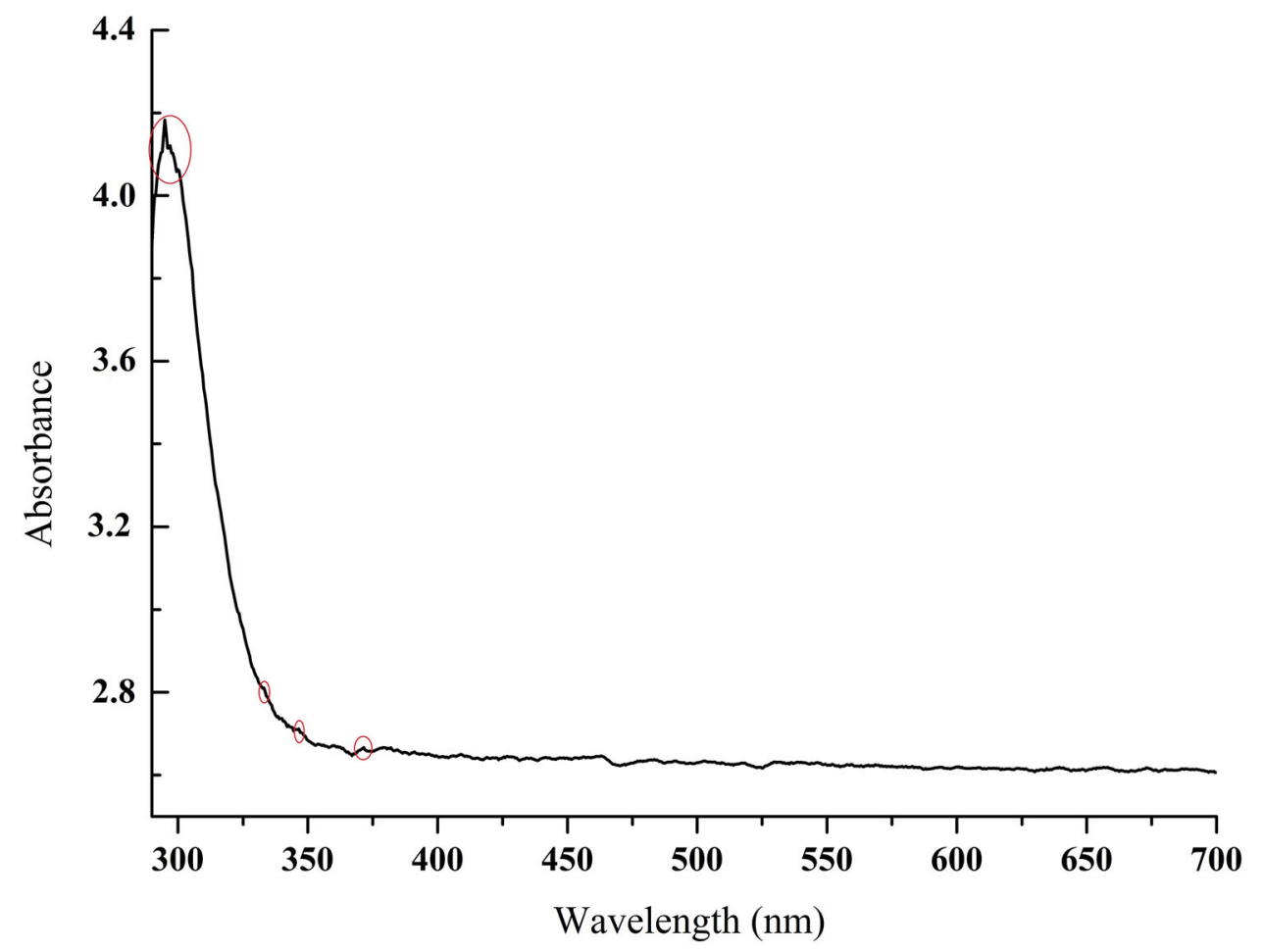

Figure 7. UV-vis analysis of graphene-BN nanosheet heterostructures.

at the end of the milling process was removed from the structure during acid treatment and thermal process and structures with stable sheets remained. TGA analysis was conducted on milled $\mathrm{h}-\mathrm{G}$ and $\mathrm{h}-\mathrm{BN}$ mixed in acid and exposed to a thermal process in order to determine the amorphous nature. Amorphous structures formed with ball impacts during the milling process were active because they contained several fractured bonds. They burned due to the effect of oxygen and high temperature. Figure 6 shows the TGA results obtained. Figure $6 a$ shows TGA analysis of $\mathrm{h}-\mathrm{G}$ at the end of the milling process. The results similar to this analysis were also obtained in our previous study [18]. Figure 6b shows TGA analysis of the milled h-BN.

While there was $65 \%$ substance loss in milled h-G, substance loss of milled h-BN was about $17 \%$. Substance loss occurring as a result of TGA analysis was due to burned amorphous structures. Graphite and BN in the crystal form found in milled powders did not burn during TGA. Figure $6 \mathrm{c}$ shows TGA analysis of the sample obtained after milled $\mathrm{h}-\mathrm{G}$ and $\mathrm{h}-\mathrm{BN}$ powders were mixed in acid solution. As is seen from the figure, there was substance loss again and the loss was relatively less. Figure $6 \mathrm{~d}$ shows TGA analysis of the sample subjected to the thermal process following acid treatment. As is seen, there was no substance loss in the sample where distance between sheets was expanded. This indicated that amorphous structures were substantially removed during this processes and crystal graphite and $\mathrm{BN}$ were dominant in the structure. Raman spectrum also confirms this. Some of amorphous structures were considered to dissolve in acid mixing performed after the milling process. A great amount of amorphous structures remaining due to the thermal process that was carried out after acid treatment were estimated to burn.

Figure 7 shows UV-vis spectrometer analysis result of graphene-BN nanosheet heterostructures. A strong peak at $295 \mathrm{~nm}$ and some weak peaks at 323, 339 and $370 \mathrm{~nm}$ are observed in the absorption spectrum, which are assigned to the optical band gap of h-BN (strong peak) and $\pi$-plasmon absorption of graphene (weak peak) [26].

\section{Conclusions}

The liquid exfoliation method assisted by a milling process was preferred for production of graphene-BN nanosheet heterostructures. Applying a milling process in which shear stresses were effective to powders of graphite and BN with hexagonal structures allowed to become sheets with smaller size by separating sheets. As we expected, amorphization occurred in powders at the end of the milling process. The stratified structure remaining after mixing milled powders was subjected to a two-stage process in order to increase distance between the sheets. This stage including acid treatment and thermal process did not allow the distance between sheets to increase and also ensured to remove amorphous structures completely. Powders obtained from this process were sonicated in DMF. Consequently, graphene-BNNS 
heterostructures were successfully synthesized. Structures except for nanosheets were also found in the structure even at a very low amount. These were thought to be amorphous structures in small quantities remaining from the milling process. Thick sheets were not found in the sample.

\section{Acknowledgements}

We would like to acknowledge the financial support from Mersin University Department of Scientific Research Projects (Project No. 2017-2-AP4-2560).

\section{References}

[1] Singh V, Joung D, Zhai L, Das S, Khondaker S I and Seal S 2011 Prog. Mater. Sci. 561178

[2] Güler Ö, Güler S H, Selen V, Albayrak M G and Evin E 2016 Fuller. Nanotubes Carbon Nanostruct. 24123

[3] Selvam M, Sakthipandi K, Suriyaprabha R, Saminathan K and Rajendran V 2013 Bull. Mater. Sci. 361315

[4] Novoselov K S, Geim A K, Morozov S V, Jiang D, Zhang Y, Dubonos S V et al 2004 Science 306666

[5] Geim A K and Novoselov K S 2007 Nat. Mater. 6183

[6] Zhao X, Zhang Q, Chen D and Lu P 2010 Macromolecules 43 2357

[7] Cui X, Zhang C and Hao Hou Y 2011 Nanoscale 32118

[8] Guo J M, Gao H L and Qin X 2015 Fuller. Nanotubes Carbon Nanostruct. 23477

[9] Chen M L, Park C Y, Meng Z D, Zhu L, Choi J G, Ghosh T et al 2013 Fuller. Nanotubes Carbon Nanostruct. 21525
[10] Yurdakul H, Göncü Y, Durukan O, Akay A, Seyhan A T, Ay N et al 2012 Ceram. Int. 382187

[11] Alem N, Erni R, Kisielowski C, Rossell M D, Gannett W and Zettl A 2009 Phys. Rev. B 80155425

[12] Golberg D, Bando Y, Huang Y, Terao Y, Mitome M, Tang C et al 2010 ACS Nano 42979

[13] Gao R, Yin L, Wang C, Qi Y, Lun N, Zhang L et al 2009 J. Phys. Chem. C 11315160

[14] Ishigami M, Chen J H, Cullen W G, Fuhrer M S and Williams E D 2007 Nano Lett. 71643

[15] Hwang E H, Adam S and Sarma S D 2007 Phys. Rev. Lett. 98 186806

[16] Chen J H, Jang C, Xiao S, Ishigami M and Fuhrer M S 2008 Nat. Nanotechnol. 3206

[17] Dean C R, Young A F, Meric I, Lee C, Wang L, Sorgenfrei S et al 2010 Nat. Nanotechnol. 5722

[18] Güler S H, Güler Ö and Evin E 2017 Fuller. Nanotubes Carbon Nanostruct. 2534

[19] Güler Ö and Güler S H 2016 Optik Int. J. Light Electron Opt. 1274630

[20] Dhakate S R, Chauhan N, Sharma S, Tawale J, Singh S, Sahare P D et al 2011 Carbon 491946

[21] Liu C Q, Hu G X and Gao H Y 2012 J. Supercrit. Fluids 63 99

[22] Zhu L X, Zhao X, Li Y Z, Yu X Y, Li C and Zhang Q H 2013 Mater. Chem. Phys. 137984

[23] Guler O and Evin E 2015 Fuller. Nanotubes Carbon Nanostruct. 23463

[24] Lin Y, Williams T V and Connell J W 2010 J. Phys. Chem. Lett. 1277

[25] Yu J, Qin L, Hao Y, Kuang S, Bai X, Chong Y M et al 2010 ACS Nano 4414

[26] Xu Z, Khanaki A, Tian H, Zheng H, Suja M, Zheng J G et al 2016 Appl. Phys. Lett. 10943110 